\title{
Publication comment
}

Tiit Mathiesen

Received: 8 April 2010 /Published online: 30 April 2010

(C) Springer-Verlag 2010

Case reports are important as "proof of principle". This case supposedly demonstrated that superficial siderosis may progress and lead to death in a clinical condition indistinguishable from ALS in spite of successful removal of a source of hemorrhage. This is an elegant observation and an intriguing article.

The weakness is, however, that the authors are not able to demonstrate their main point: that they have identified a cause of hemorrhage and removed it: (1) There is no preoperative image of a possible source of intradural hemorrhage. (2) There is an intraoperative observation of a "venous anomaly". A venous anomaly is usually regarded as a developmental finding that may coexist with a cavernoma, but which is itself not a likely cause of hemorrhage. (3) There is no histology to prove that the lesion is potentially hemorrhagic. (4) There are no surgical findings of previous hemorrhage around the lesion they explored (MRI superficial siderosis was in a different

T. Mathiesen $(\bowtie)$

Division of Clinical CNS Research,

Karolinska University Hospital,

Stockholm, Sweden

e-mail: tiit.mathiesen@karolinska.se location, the fluid collection was not described as xanthocromic and the images show no signs of previous hemorrhage in the surgical field). (5) The argument of "no further bleeding occurred after surgery" is weak, as we have no actual clinical or radiological evidence of repeated hemorrhages before surgery, just the clinical presentation of progressive neurological deficits and one image of intracranial superficial signal abnormalities. It is thus not stated how episodes of bleeding before and after surgery were defined or assessed.

In addition, they have not ruled out the possibility that the patient actually died from amyotrophic lateral sclerosis (ALS). ALS is not an uncommon cause of death in a neurological practice. While the chance of finding a patient with coexisting ALS and SS is small, the argument of two rare conditions is not applicable if you are concerned with a patient who is already selected for a condition compatible with ALS.

The case is highly interesting and puzzling, but we lack data and knowledge that would make this case report anything but a biased, albeit interestingly written, speculation of one possible explanation of their data. The patient may well have died from superficial siderosis; such a cause of death agrees well with Occam's razor and my interpretation: surgery was an epiphenomenon. 